\title{
Precision electromagnetic calorimetry at the energy frontier: The CMS ECAL at the LHC Run 2
}

\author{
Badder Marzocchi*i \\ Università di Milano-Bicocca \& INFN Sezione di Milano-Bicocca \\ E-mail: badder.marzocchi@cern. ch
}

\begin{abstract}
The Large Hadron Collider (LHC) has recently restarted operations (Run 2) with proton-proton collisions at an unprecedented centre-of-mass energy of $13 \mathrm{TeV}$ and is moving to a reduced bunch spacing of $25 \mathrm{~ns}$. After the successful quest for a Higgs boson via its electromagnetic decays, and the subsequent measurement of its mass, the CMS electromagnetic calorimeter (ECAL) plans to perform precision measurements and searches for new physics phenomena beyond the standard model with the data that are being collected. In this poster we present new reconstruction algorithms and calibration strategies that aim to maintain, and even improve, the excellent performance of the CMS ECAL under the new challenging experimental conditions. The CMS ECAL is a high-resolution, hermetic, and homogeneous electromagnetic calorimeter made of 75,848 scintillating lead tungstate crystals. Its resolution, as well as its timing performance, are valuable tools for the discovery of new physics with the CMS detector at the LHC. The performance of the calorimeter relies on precision calibration maintained over time, despite severe irradiation conditions. A set of intercalibration procedures using different physics channels is carried out at regular intervals to normalize the differences in crystal light transparency and photodetector response between channels, which can change due to accumulated radiation.
\end{abstract}

The European Physical Society Conference on High Energy Physics

22-29 July 2015

Vienna, Austria

\footnotetext{
* Speaker.

${ }^{\dagger}$ On behalf of CMS Collaboration
} 


\section{ECAL detector}

During the first period of LHC operation (Run 1), the CMS electromagnetic calorimeter (ECAL) [1] played an essential role in the search for the Higgs boson by measuring with high precision the energy of photons arising from $\mathrm{H} \rightarrow \gamma \gamma$ decays and of electrons from $\mathrm{H} \rightarrow \mathrm{ZZ}$ decays. To achieve this, a homogeneous and hermetic calorimeter, made of scintillating lead tungstate crystals, has been designed. This material is suitable for operation at LHC due to its fast emission ( $80 \%$ of the scintillation light is emitted within $25 \mathrm{~ns}$ ) and its resilience to irradiation. Moreover, thanks to crystals' short radiation length $\left(\mathrm{X}_{0}=0.89 \mathrm{~cm}\right)$ and small Molière radius $\left(r_{M}=21.9 \mathrm{~mm}\right)$, most of an electron or photon's energy can be collected within a small matrix of crystals. The relatively low light yield of $\simeq 30 \gamma / \mathrm{MeV}$ makes it necessary to use intrinsic high-gain photodetectors, capable of operating in high magnetic fields. Avalanche PhotoDiodes (APDs) are used for the barrel crystals and Vacuum PhotoTriodes (VPTs) are used for the endcaps crystals.

As shown in Figure 1, ECAL is divided in two main parts:
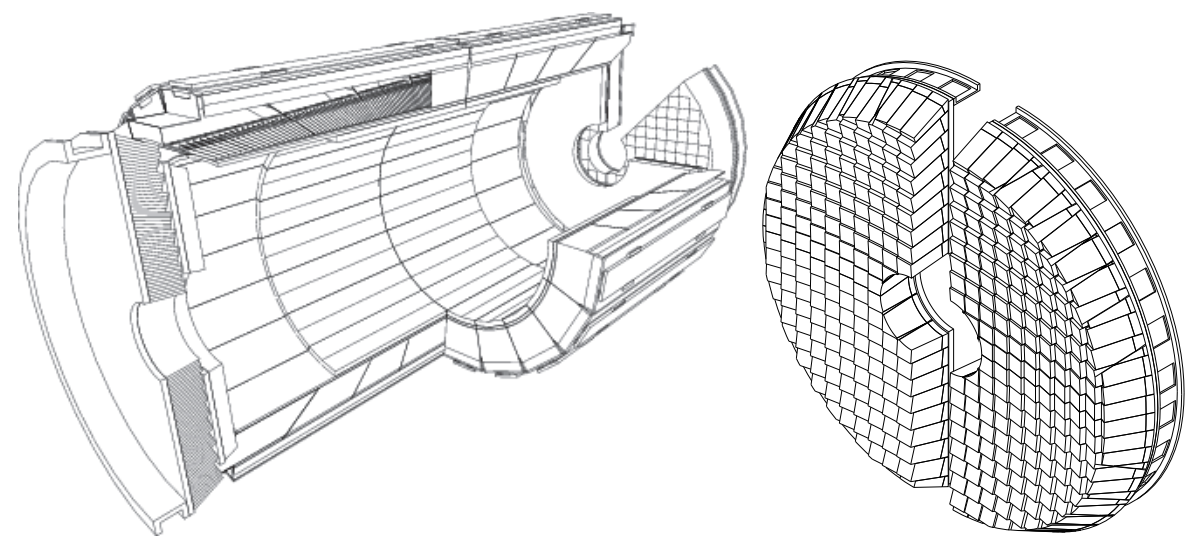

Figure 1: CMS ECAL geometry schema. The ECAL barrel (EB) is made of 36 SuperModules: 18 in EB+ $(z>0)$ and 18 in EB- $(z<0)$ as depicted in (a). The ECAL endcap (EE) is divided in 4 Dees: 2 Dees in EE+ $(z>0)$ and 2 Dees in EE+ $(z>0)$ as depicted in $(b)$.

- Barrel (EB): it is made of 61200 crystals and it is shaped as a cylinder, with an inner radius of $1.290 \mathrm{~m}$. It covers a pseudorapidity range of $0<|\eta|<1.479$ and its granularity is 360 crystals in $\phi$ and $2 \times 85$ crystals in $\eta$. Crystals in EB are mounted in a quasi-projective geometry to avoid cracks aligned with particle trajectories, so that the crystal axes are tilted at an angle of $3^{\circ}$ in both the $\phi$ and $\eta$ projections.

- Two endcaps (EE): they cover the pseudorapidity range $1.479<|\eta|<3.0$ and consist of identically shaped crystals, grouped into carbon-fiber structures of $5 \times 5$ elements, called supercrystals. Each endcap is divided into 2 halves, or Dees, holding 3662 crystals each.

In addition, a preshower (ES) is placed in front of EE crystals with the aim of identifying neutral pions in the endcaps and improving the position determination of electrons and photons. The preshower consists of two layers made of passive lead radiators $\left(2 X_{0}\right.$ and $\left.1 X_{0}\right)$, which initiate electromagnetic showers, and active silicon strip sensors placed after each radiator (4288 strips, 
thickness of $310 \mu \mathrm{m}$ and area of $1.9 \mathrm{~mm} \times 61 \mathrm{~mm}$ ), which measure the deposited energy and the transverse shower profiles.

\section{ECAL performance during Run 1}

When a photon or an electron strikes the detector it produces an electromagnetic shower in the crystals. The energy of the electromagnetic showers is deposited in crystals matrices. Furthermore the 3.8 T CMS magnetic field causes the energy deposition to be spread along $\phi$. Clustering algorithms are used to sum together energy deposits in adjacent crystals belonging to the same electromagnetic shower. The clustering algorithm begins first with the formation of "basic clusters", corresponding to local maxima of energy deposits. The basic clusters are then merged together to form a "supercluster", which is extended in $\phi$, to recover the radiated energy.

During Run 1 data-taking, the ECAL detector fully achieved the requirements for electron [2] and photon [3] identification. In particular, the excellent photon resolution was a critical ingredient in the observation of the Higgs boson in the $\mathrm{H} \rightarrow \gamma \gamma$ channel and the precision measurement of its properties [4]. The energy resolution is optimized using simulated electrons of photons and it is verified in data reconstructing electron showers from $\mathrm{Z} \rightarrow e e$ as photons, in two $\mathrm{R}_{9}$ regions, as shown in Figure 2. $R_{9}$ is defined as the energy sum of the $3 \times 3$ crystals centred on the most energetic crystal in the supercluster divided by the energy of the supercluster. Converted photons or showering electrons have wider transverse profiles and lower values of $R_{9}$.
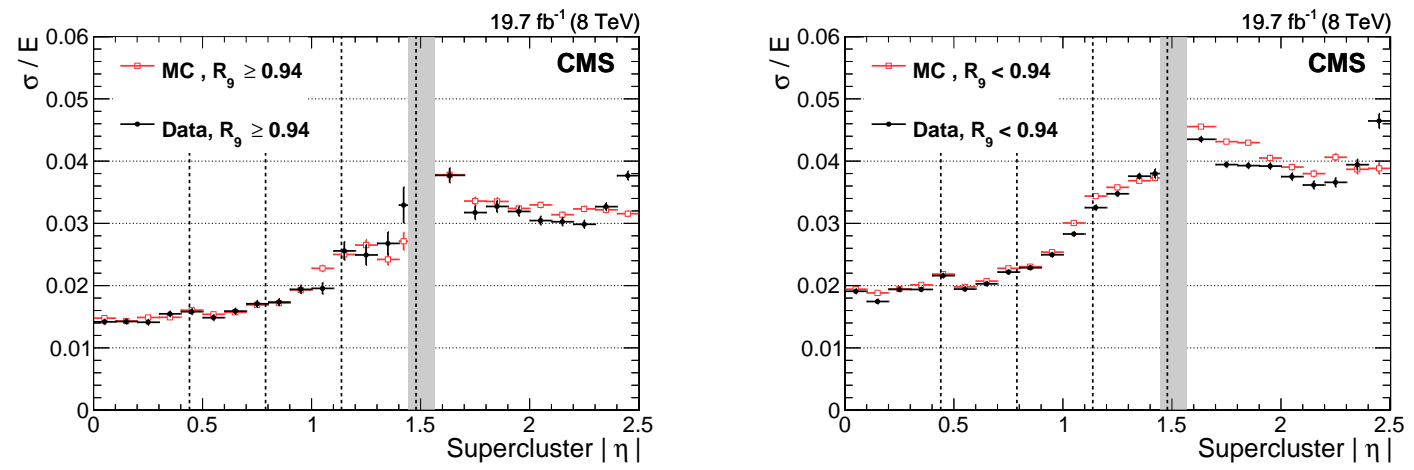

Figure 2: Relative photon energy resolution measured in bins of absolute supercluster pseudorapidity in $\mathrm{Z} \rightarrow e e$ events, for data (solid black circles) and simulated events (open squares), where the electrons are reconstructed as photons. The simulation is tuned with observed data $\mathrm{Z} \rightarrow e e$ events. The resolution is shown for showers with $\mathrm{R}_{9}>0.94$ (left) and $\mathrm{R}_{9}<0.94$ (right).

\section{Energy calibration}

In Run 1 and Run 2, the energy reconstruction and calibration procedures are similar. The main difference is related to the way the amplitude of the single crystals pulse-shapes is reconstructed. 
The energy in a supercluster can be expressed as:

$$
E_{e, \gamma}=F_{e, \gamma} \cdot\left[G \cdot \sum_{i} S_{i}(t) \cdot C_{i} \cdot A_{i}+E_{E S}\right]
$$

where the sum runs over all the crystals composing the supercluster, $E_{E S}$ is the fraction of energy measured in the preshower and $A_{i}$ is the ADC amplitude.

- $C_{i}$ : the intercalibration term, which takes into account crystal-to-crystal variations in the response by equalizing the different crystal responses.

- $S_{i}(t)$ : the time dependent transparency correction.

- $G$ : the $\mathrm{ADC}$ to $\mathrm{GeV}$ conversion factor, determined using $\mathrm{Z} \rightarrow \mathrm{e}^{+} \mathrm{e}^{-}$events.

- $F_{e, \gamma}$ : energy corrections, applied to the superclusters to take into account the $\eta$ and $\phi$ dependent geometry and material effects as well as the fact that electrons and photons shower slightly differently.

\subsection{Amplitude reconstruction}

For each crystal in EB and EE ten consecutive samples are stored for each trigger received. The signal pulse is expected to start from the fourth sample and the baseline pedestal can be estimated from the first three samples. During Run 1 the amplitude of the signal was reconstructed using a linear combination of the samples:

$$
A=\sum_{j} w_{j} \cdot s_{j}
$$

where $s_{j}$ is the sample value in ADC counts, $w_{j}$ is a weight and $j$ runs over the samples. $w_{j}$ is computed using a " $3+5$ pedestal-subtracting weights" method, optimized for noise reduction [5]. The conditions that will be experienced during LHC Run 2 place particular requirements on the ECAL pulse reconstruction algorithms. The number of inelastic collisions per LHC bunch crossing (pileup) will increase. In addition, the spacing between colliding bunches has been reduced from $50 \mathrm{~ns}$ to $25 \mathrm{~ns}$ in 2015 , increasing the level of the out-of-time pileup, i.e. spurious contribution to the amplitude coming from the out-of-time bunch crossings. Because of these conditions, a new reconstruction method, which is less sensitive to the pile-up, has been developed. The algorithm estimates the signal amplitude with the addition of up to 9 pulse shapes every $25 \mathrm{~ns}$ by the minimization of the $\chi^{2}$, given by:

$$
\chi^{2}=\sum_{i}^{N} \frac{\left(\sum_{j}^{M} A_{j} p_{i j}-S_{i}\right)^{2}}{\sigma_{S_{i}}^{2}}
$$

where $A_{j}$ are the amplitudes, $p_{i j}$ are the pulses (all identical in shape, shifted by $25 \mathrm{~ns}$ ) and $\sigma_{S_{i}}^{2}$ is the noise covariance matrix. Figure 3 shows examples of fitted pulses for simulated events with 20 average pileup interactions and $25 \mathrm{~ns}$ bunch spacing, for a signal in the barrel and in the endcaps using this reconstruction method. According to the simulation, this method provides an improved resilience to pile-up, in particular for electromagnetic showers of low energy. 

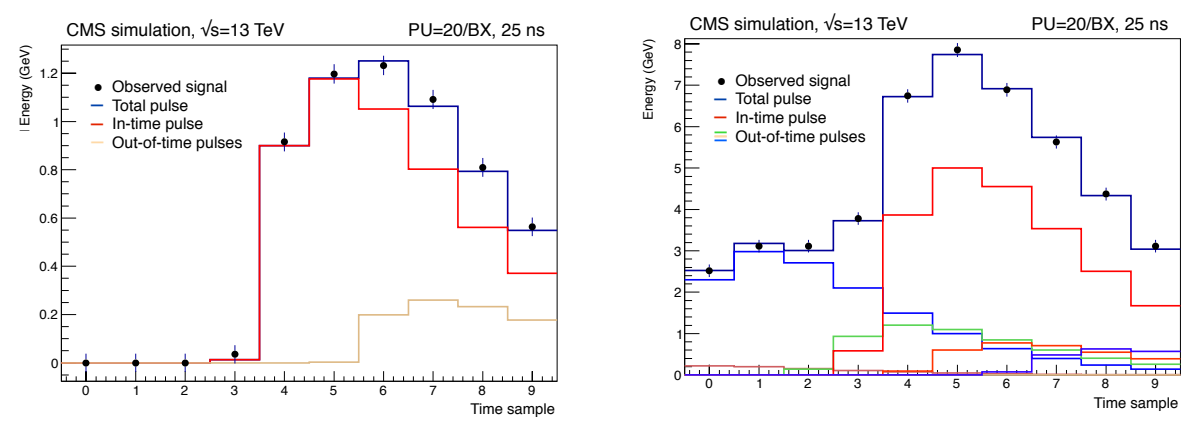

Figure 3: Example of fitted pulses for simulated events with 20 average pileup interactions and $25 \mathrm{~ns}$ bunch spacing, for a signal in the barrel (left) and in the endcaps (right). Dots represent the 10 digitized samples, the red distributions (other light colours) represent the fitted in-time (out-of time) pulses with positive amplitude. The dark blue histograms represent the sum of all the fitted contributions.

\subsection{Time dependent corrections}

The optical transmission of the $\mathrm{PbWO}_{4}$ crystals at the scintillation wavelengths is affected by the production of colour centres under ionizing radiation. This causes a non-permanent transparency loss. In fact spontaneous annealing of the colour centres occurs also at room temperature and leads to a transmission recovery, which is evident when the crystals are not irradiated. Uncertainties in the relative measurement of the optical transmission from crystal to crystal contribute directly to the energy resolution. In situ light transmission measurements are performed through a laser monitoring system which is based on injecting light into each crystal.

The energy correction factor extracted by means of the laser monitoring system depends on the light collection mechanisms of both electromagnetic showers $\left(S / S_{0}\right)$ and injected laser $\left(R / R_{0}\right)[6]$ :

$$
\frac{S}{S_{0}}=\left(\frac{R}{R_{0}}\right)^{\alpha}
$$

where $\alpha$ is an empirical parameter.

In the endcaps the absorbed radiation dose is much higher than in the barrel; therefore during the LHC running VPTs response can deteriorate, leading to an inaccurate $R / R_{0}$ measurement and affecting the measurement of the $\alpha$ value.

Figure 4 shows the in situ measurement of the transparency loss during 2011, 2012 and the beginning of 2015 data-taking for different $\eta$ regions. The recovery of the crystals during the LHC shut-downs is evident. However the response is not fully recovered, giving an indication of a permanent damage of crystals and photodetectors, especially in the endcaps.

\subsection{Single-channel intercalibration}

The channel by channel intercalibration [7] involves many methods: $\pi^{0} / \eta$, isolated electrons from "W" and "Z" decays and $\phi$-symmetry. All these methods are used to intercalibrate channels at the same pseudorapidity. The precision of the intercalibrations has been studied for each method with the aid of Monte Carlo simulations and by a cross comparison of the calibration coefficients derived by each method: 


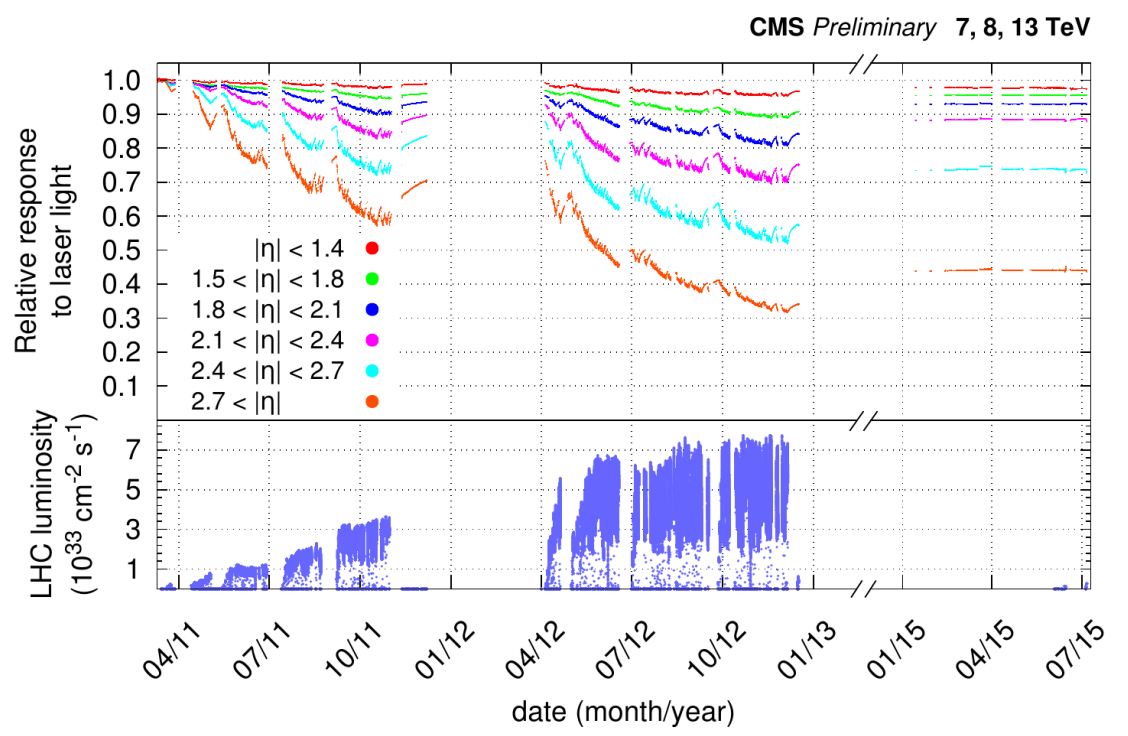

Figure 4: Relative response to laser light (440 nm in 2011 and $447 \mathrm{~nm}$ from 2012 onwards) injected in the ECAL crystals, measured by the ECAL laser monitoring system, averaged over all crystals in bins of pseudorapidity, for the 2011, 2012 and early 2015 data-taking periods. These measurements are used to correct the physics data. The bottom plot shows the instantaneous LHC luminosity delivered during this time period.

- $\pi^{0} / \eta$-calibration: iterative method exploiting the invariant mass reconstructed from unconverted photons arising from the decays of $\pi^{0} / \eta$. A precision of $0.5 \%$ in the central barrel (dominated by the systematics uncertainties) is foreseen for the Run 2.

- E/p: iterative method, based on the comparison of the ECAL energy to the tracker momentum for isolated electrons. In central barrel the precision reaches the systematic limit of $0.5 \%$. For $|\eta|>1$ the statistical contribution was the limiting factor for Run 1, whereas more statistic is expected for the Run 2.

- $\phi$-symmetry: based on the equalization of the average energy in channels located at a constant value of $\eta$. The accuracy is limited to a few percent by systematic uncertainties in the distribution of material in front of the ECAL.

Figure 5 shows the precision of different intercalibration procedures and the final combination from Run 1 , as a function of $\eta$, for the barrel and the endcaps.

\section{Run 2 preliminary results}

During the first $21.3 \mathrm{pb}^{-1}$ of data collected in 2015 , the calibration constants extracted from the 2012 data have been used for a preliminary calibration of the channel response. In addition, the response of each channel has been corrected by the response monitoring system, as shown in Figure 4. On the other hand concerning the Monte Carlo simulation, the asymptotic precision of 

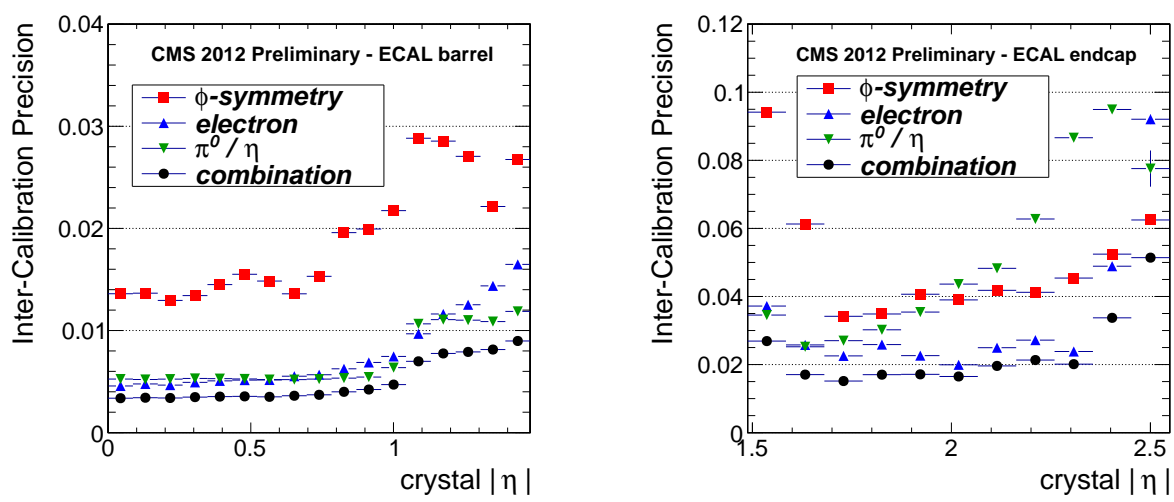

Figure 5: Precision for measuring the intercalibration constants from $\phi$-symmetry, from $\pi^{0} \rightarrow \gamma \gamma$ and $\eta \rightarrow$ $\gamma \gamma$ decays, and from $\mathrm{W}$ and $\mathrm{Z}$ decay electrons, is shown as a function of $\eta$ in EB (left) and EE (right), using 2012 data. The black points represent the precision of the combination of the three methods (weighted average).

calibration with Run 2 conditions has been injected for the reconstruction. Figure 6 shows preliminary results on the reconstructed $\mathrm{Z} \rightarrow e e$ invariant mass for the first $13 \mathrm{TeV}$ data and Monte Carlo simulation in EB and in EE. Although the agreement will be improved applying intercalibrations derived from 2015 data, the current precision gives enough sensitivity to the first analyses, such as $\mathrm{W}^{\prime}$ or $\mathrm{Z}^{\prime}$ searches.
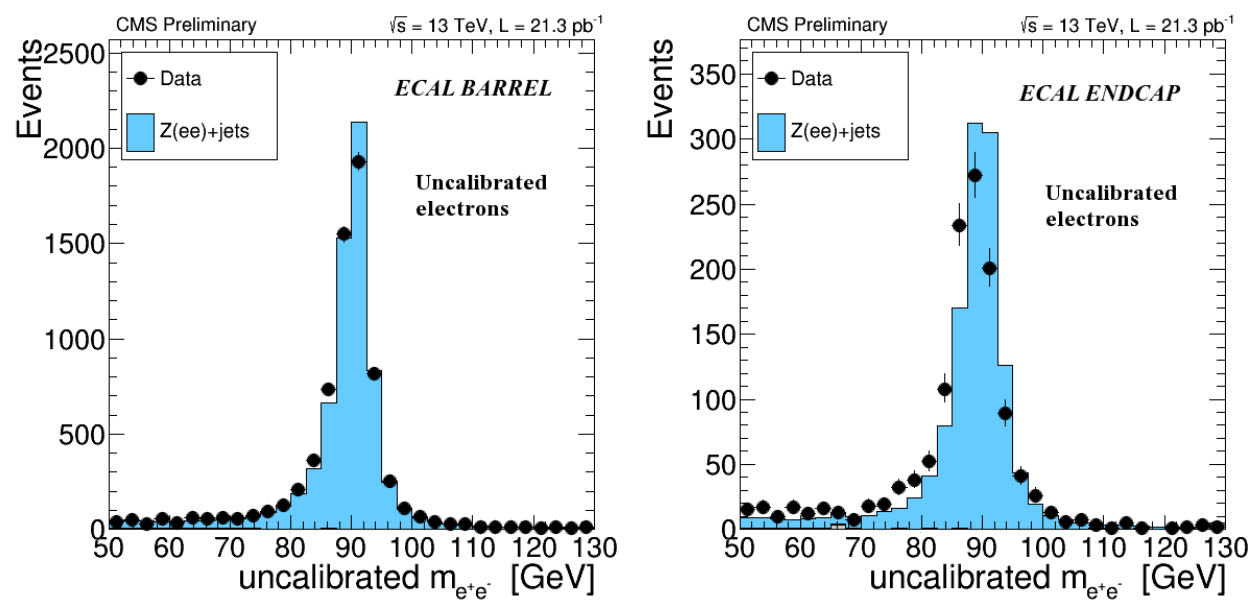

Figure 6: $\mathrm{Z} \rightarrow e e$ invariant mass plot for 2015 data, from the reconstruction of dielectron events with both electrons in the ECAL Barrel (left) and both electrons in the ECAL Endcap (right). The calibrations applied to these early 50ns Run 2 data are the Run 1 ones, extrapolated in time using the laser measurements only. The simulation is not tuned in terms of the noise and pileup observed in this dataset, and assumes a calibration precision that will be achieved at the end of the 50ns LHC run (not yet applied to the data)

\section{Conclusions}

A description of the particles' reconstruction and calibration, performed with the CMS ECAL 
detector, is presented for the first Run 2 data. In addition, preliminary results using $\mathrm{Z} \rightarrow e e$ events are shown.

\section{References}

[1] CMS Collaboration, "The CMS electromagnetic calorimeter project: Technical Design Report", CERN-LHCC-97-033

[2] CMS Collaboration, "Performance of electron reconstruction and selection with the CMS detector in proton-proton collisions at $\sqrt{s}=8$ TeV", JINST 10 (2015) P06005

[3] CMS Collaboration, "Performance of photon reconstruction and identification with the CMS detector in proton-proton collisions at $\sqrt{s}=8$ TeV", JINST 10 (2015) P08010

[4] CMS Collaboration, "Observation of the diphoton decay of the Higgs boson and measurement of its properties", Eur. Phys. J. C 74 (2014) 3076

[5] P. Adzic, et al., "Reconstruction of the signal amplitude of the CMS electromagnetic calorimeter", CERN-CMS-NOTE-2006-037

[6] Anfrevilleb M. et al., "Laser monitoring system for the CMS lead tungstate crystal calorimeter", CMS-NOTE-2007-028,

[7] CMS Collaboration, "Energy calibration and resolution of the CMS electromagnetic calorimeter in pp collisions at $\sqrt{s}=7$ TeV", JINST 8 (2013) P09009 